\title{
MAGNETOHYDRODYNAMICS EFFECT ON THREE-DIMENSIONAL VISCOUS INCOMPRESSIBLE FLOW BETWEEN TWO HORIZONTAL PARALLEL POROUS PLATES AND HEAT TRANSFER WITH PERIODIC INJECTION/SUCTION
}

\author{
PAWAN KUMAR SHARMA and R. C. CHAUDHARY
}

Received 23 November 2003

\begin{abstract}
We investigate the hydromagnetic effect on viscous incompressible flow between two horizontal parallel porous flat plates with transverse sinusoidal injection of the fluid at the stationary plate and its corresponding removal by periodic suction through the plate in uniform motion. The flow becomes three dimensional due to this injection/suction velocity. Approximate solutions are obtained for the flow field, the pressure, the skin-friction, the temperature field, and the rate of heat transfer. The dependence of solution on $M$ (Hartmann number) and $\lambda$ (injection/suction) is investigated by the graphs and tables.
\end{abstract}

2000 Mathematics Subject Classification: 76D99, 76W05, 80A20.

1. Introduction. The problem of laminar flow control has become very important in recent years, particularly in the field of aeronautical engineering, owing to its application in reducing drag and hence in enhancing the vehicle power by a substantial amount. Several methods have been developed for the purpose of artificially controlling the boundary layer and the developments on this subject. The boundary layer suction is one of the effective methods of reducing the drag coefficient, which entails large energy losses. The effect of different arrangements and configurations of the suction holes and slits has been studied by various scholars. Gersten and Gross [2] have investigated the flow and heat transfer along a plane wall with periodic suction velocity. Effects of such a suction velocity on various flow and heat transfer problems along flat and vertical porous plates have been studied by Singh et al. [10, 11] and Singh [8]. Recently the problem of transpiration cooling with the application of the transverse sinusoidal injection/suction velocity has been studied by Singh [9].

Magnetic fields influence many natural and man-made flows. They are routinely used in industry to heat, pump, stir, and levitate liquid metals. There are the terrestrial magnetic field, which is maintained by fluid motion in the earth's core, the solar magnetic field which generates sunspots and solar flares, and the galactic field which influences the formation of stars. The flow problems of an electrically conducting fluid under the influence of magnetic field have attracted the interest of many authors in view of their applications to geophysics, astrophysics, engineering, and to the boundary layer control in the field of aerodynamics. On the other hand, in view of the increasing technical applications using magnetohydrodynamics (MHD) effect, it is desirable to extend many of the available viscous hydrodynamic solutions to include the effects of magnetic field 
for those cases when the viscous fluid is electrically conducting. Rossow [5], Greenspan and Carrier [3], and Singh [6, 7] have studied extensively the hydromagnetic effects on the flow past a plate with or without injection/suction. The hydromagnetic channel flow and temperature field was investigated by Attia and Kotb [1]. Hossain et al. [4] have studied the MHD free convection flow when the surface is kept at oscillating surface heat flux. Boundary layer flows of fluids of small electrical conductivity are important, particularly in the field of aeronautical engineering. Therefore the object of the present note is to study the effects of the magnetic field on the flow of a viscous, incompressible, and electrically conducting fluid between two horizontal parallel porous plates with transverse sinusoidal injection of the fluid at the stationary plate and its corresponding removal by periodic suction through the plate in uniform motion.

2. Formulation of the problem. We consider the Couette flow of a viscous incompressible electrically conducting fluid between two parallel flat porous plates with transverse sinusoidal injection of the fluid at the stationary plate and its corresponding removal by periodic suction through the plate in uniform motion $U$. Let the $x^{*}-z^{*}$ plane lie along the plates and let the $y^{*}$-axis be taken normal to the free-stream velocity. The distance $d$ is taken between the plates. Denote the velocity components by $u^{*}, v^{*}, w^{*}$ in the $x^{*}-, y^{*}-, z^{*}$-directions, respectively. The lower and upper plates are assumed to be at constant temperature $T_{0}$ and $T_{1}$, respectively, with $T_{1}>T_{0}$. We derive the governing equations with the assumption that the flow is steady and laminar, and is of a finitely conducting fluid. The magnetic field of uniform strength $B$ is applied to the perpendicular of the free-stream velocity (see Figure 2.1); at lower magnetic Reynolds number, the magnetic field is practically independent of the flow motion and the induced magnetic field is neglected. The Hall effects, electrical and polarization effects also have been neglected. All physical quantities are independent of $x^{*}$ for this problem of fully developed laminar flow, but the flow remains three-dimensional due to the injection/suction velocity $\mathbf{V}^{*}\left(\mathbf{z}^{*}\right)=\mathbf{V}\left(\mathbf{1}+\varepsilon \cos \pi \mathbf{z}^{*} / \mathbf{d}\right)$. Thus, under these assumptions, the problem is governed by the following nondimensional system of equations:

$$
\begin{gathered}
v_{y}+w_{z}=0, \\
v u_{y}+w u_{z}=\frac{\left[u_{y y}+u_{z z}-M^{2}(u-1)\right]}{\lambda}, \\
v v_{y}+w v_{z}=-p_{y}+\frac{\left[v_{y y}+v_{z z}\right]}{\lambda}, \\
v w_{y}+w w_{z}=-p_{z}+\frac{\left[w_{y y}+w_{z z}-M^{2} w\right]}{\lambda}, \\
v \theta_{y}+w \theta_{z}=\frac{\left(\theta_{y y}+\theta_{z z}\right)}{\lambda \operatorname{Pr}},
\end{gathered}
$$

where $y=y^{*} / d, z=z^{*} / d, u=u^{*} / U, v=v^{*} / V, w=w^{*} / V, p=p^{*} / \rho V^{2}$, Pr (Prandtl number) $=v / \alpha, M$ (Hartmann number) $=\left(\sigma B^{2} d^{2} / \rho v\right)^{1 / 2}, \lambda$ (injection/suction parameter $)=V d / \nu$, and $\theta=\left(T^{*}-T_{0}\right) /\left(T_{1}-T_{0}\right)$ are the dimensionless quantities and $V, \rho, \nu, \alpha, \sigma$, and $p$ are, respectively, injection/suction velocity, density, kinematic 


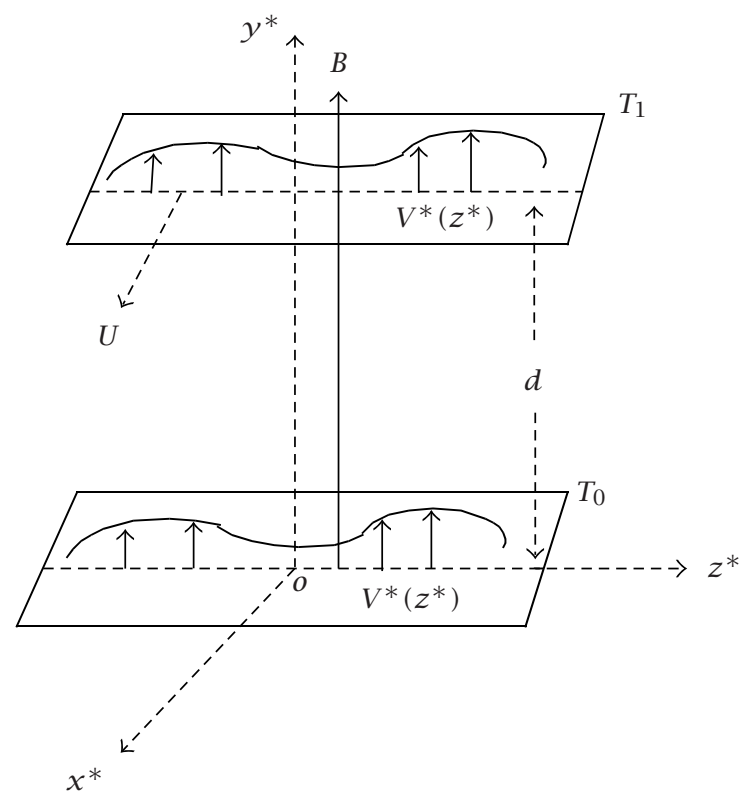

FIGURE 2.1. Schematic of the flow configuration.

viscosity, thermal diffusivity, electrical conductivity, and pressure. In energy equation terms corresponding to viscous dissipation and Joule heating have been neglected due to their small magnitude as compared to other terms. The $\left(^{*}\right)$ stands for dimensional quantities. The corresponding boundary conditions in the dimensionless form are

$$
\begin{array}{llll}
y=0: u=0, & v(z)=1+\varepsilon \cos \pi z, & w=0, & \theta=0, \\
y=1: u=1, & v(z)=1+\varepsilon \cos \pi z, & w=0, & \theta=1 .
\end{array}
$$

3. Solution of the problem. Since the amplitude of the injection/suction velocity $\varepsilon(\ll 1)$ is very small, we now assume the solution of the following form:

$$
f(y, z)=f_{0}(y)+\varepsilon f_{1}(y, z)+\varepsilon^{2}(y, z)+\cdots,
$$

where $f$ stands for any of $u, v, w, p$, and $\theta$. When $\varepsilon=0$, the problem is reduced to the well-known two-dimensional flow. The solution of this two-dimensional problem is

$$
\begin{gathered}
u_{0}(y)=1+\frac{\left[\exp \left(J_{2}+J_{1} y\right)-\exp \left(J_{1}+J_{2} y\right)\right]}{\left[\exp \left(J_{1}\right)-\exp \left(J_{2}\right)\right]} \\
\theta_{0}(y)=\frac{[\exp (\lambda \operatorname{Pr} y)-1]}{[\exp (\lambda \operatorname{Pr})-1]}, \quad v_{0}=1, \quad w_{0}=0, \quad p_{0}=\text { constant, }
\end{gathered}
$$


where

$$
J_{1}=\frac{\left[\lambda+\left(\lambda^{2}+4 M^{2}\right)^{1 / 2}\right]}{2}, \quad J_{2}=\frac{\left[\lambda-\left(\lambda^{2}+4 M^{2}\right)^{1 / 2}\right]}{2} .
$$

When $\varepsilon \neq 0$, substituting (3.1) in (2.1)-(2.5) and comparing the coefficient of $\varepsilon$, neglecting those of $\varepsilon^{2}, \varepsilon^{3}, \ldots$, the following first-order equations are obtained with the help of solution (3.2):

$$
\begin{gathered}
v_{1 y}+w_{1 z}=0, \\
v_{1} u_{0 y}+u_{1 y}=\frac{\left(u_{1 y y}+u_{1 z z}-M^{2} u_{1}\right)}{\lambda}, \\
v_{1 y}=-p_{1 y}+\frac{\left(v_{1 y y}+v_{1 z z}\right)}{\lambda}, \\
w_{1 y}=-p_{1 z}+\frac{\left(w_{1 y y}+w_{1 z z}-M^{2} w_{1}\right)}{\lambda}, \\
v_{1} \theta_{0 y}+\theta_{1 y}=\frac{\left(\theta_{1 y y}+\theta_{1 z z}\right)}{\lambda \operatorname{Pr}} .
\end{gathered}
$$

The corresponding boundary conditions reduce to

$$
\begin{array}{llll}
y=0: u_{1}=0, & v_{1}=\cos \pi z, & w_{1}=0, & \theta_{1}=0, \\
y=1: u_{1}=0, & v_{1}=\cos \pi z, & w_{1}=0, & \theta_{1}=0 .
\end{array}
$$

This is the set of linear partial differential equations, which describe the threedimensional flow. To solve these equations, we assume $v_{1}, w_{1}, p_{1}, u_{1}$, and $\theta_{1}$ of the following form:

$$
\begin{aligned}
& u_{1}(y, z)=u_{11}(y) \cos \pi z, \\
& v_{1}(y, z)=v_{11}(y) \cos \pi z, \\
& w_{1}(y, z)=-\frac{\left\{v_{11}^{\prime}(y) \sin \pi z\right\}}{\pi}, \\
& p_{1}(y, z)=p_{11}(y) \cos \pi z \\
& \theta_{1}(y, z)=\theta_{11}(y) \cos \pi z
\end{aligned}
$$

where the prime denotes differentiation with respect to $y$. Expressions for $v_{1}(y, z)$ and $w_{1}(y, z)$ have been chosen so that the equation of continuity (3.4) is satisfied. Substituting (3.10) in (3.5)-(3.8) and applying the corresponding boundary conditions, 
we get the solutions for $v_{1}, w_{1}, p_{1}, u_{1}$, and $\theta_{1}$ as follows:

$$
\begin{aligned}
& v_{1}(y, z)=(A)^{-1}\left[A_{1} \exp \left(J_{3} y\right)+A_{2} \exp \left(J_{4} y\right)-A_{3} \exp \left(J_{5} y\right)-A_{4} \exp \left(J_{6} y\right)\right] \cos \pi z \\
& w_{1}(y, z)=-(\pi A)^{-1}\left[A_{1} J_{3} \exp \left(J_{3} y\right)+A_{2} J_{4} \exp \left(J_{4} y\right)-A_{3} J_{5} \exp \left(J_{5} y\right)\right. \\
& \left.-A_{4} J_{6} \exp \left(J_{6} y\right)\right] \sin \pi z \\
& p_{1}(y, z)=\left(\pi^{2} \lambda A\right)^{-1}\left[A_{1} J_{3}\left(J_{1} J_{3}-\lambda J_{3}-M^{2}\right) \exp \left(J_{3} y\right)+A_{2} J_{4}\left(J_{1} J_{4}-\lambda J_{4}-M^{2}\right) \exp \left(J_{4} y\right)\right. \\
& -A_{3} J_{5}\left(J_{2} J_{5}-\lambda J_{5}-M^{2}\right) \exp \left(J_{5} y\right) \\
& \left.-A_{4} J_{6}\left(J_{2} J_{6}-\lambda J_{6}-M^{2}\right) \exp \left(J_{6} y\right)\right] \cos \pi z \\
& u_{1}(y, z)=\left[E \exp \left(g_{1} y\right)+F \exp \left(g_{2} y\right)\right. \\
& +b_{1}\left\{A_{1}\left(\frac{J_{1} \exp \left(J_{2}+J_{1} y+J_{3} y\right)}{b_{2}}-\frac{J_{2} \exp \left(J_{1}+J_{2} y+J_{3} y\right)}{b_{6}}\right)\right. \\
& +A_{2}\left(\frac{J_{1} \exp \left(J_{2}+J_{1} y+J_{4} y\right)}{b_{3}}-\frac{J_{2} \exp \left(J_{1}+J_{2} y+J_{4} y\right)}{b_{7}}\right) \\
& -A_{3}\left(\frac{J_{1} \exp \left(J_{2}+J_{1} y+J_{5} y\right)}{b_{8}}-\frac{J_{2} \exp \left(J_{1}+J_{2} y+J_{5} y\right)}{b_{4}}\right) \\
& \left.\left.-A_{4}\left(\frac{J_{1} \exp \left(J_{2}+J_{1} y+J_{6} y\right)}{b_{9}}-\frac{J_{2} \exp \left(J_{1}+J_{2} y+J_{6} y\right)}{b_{5}}\right)\right\}\right] \cos \pi z \\
& \theta_{1}(y, z)=\left[R \exp \left(s_{1} y\right)+S \exp \left(s_{2} y\right)\right. \\
& +C_{1}\left\{\frac{A_{1} \exp \left(J_{3} y+\lambda \operatorname{Pr} y\right)}{C_{2}}+\frac{A_{2} \exp \left(J_{4} y+\lambda \operatorname{Pr} y\right)}{C_{3}}\right. \\
& \left.\left.-\frac{A_{3} \exp \left(J_{5} y+\lambda \operatorname{Pr} y\right)}{C_{4}}-\frac{A_{4} \exp \left(J_{6} y+\lambda \operatorname{Pr} y\right)}{C_{5}}\right\}\right] \cos \pi z,
\end{aligned}
$$

where

$$
\begin{aligned}
A= & {\left[J_{4} J_{5}+J_{3} J_{6}-J_{3} J_{5}-J_{4} J_{6}\right]\left\{\exp \left(J_{5}+J_{6}\right)+\exp \left(J_{3}+J_{4}\right)\right\} } \\
& -\left[J_{4} J_{5}+J_{3} J_{6}-J_{3} J_{4}-J_{5} J_{6}\right]\left\{\exp \left(J_{3}+J_{5}\right)+\exp \left(J_{4}+J_{6}\right)\right\} \\
& -\left[J_{5} J_{6}+J_{3} J_{4}-J_{3} J_{5}-J_{4} J_{6}\right]\left\{\exp \left(J_{3}+J_{6}\right)+\exp \left(J_{4}+J_{5}\right)\right\}, \\
A_{1}= & {\left[J_{4} J_{6}-J_{5} J_{6}\right] \exp \left(J_{4}+J_{5}\right)-\left[J_{4} J_{5}-J_{5} J_{6}\right] \exp \left(J_{4}+J_{6}\right)+\left[J_{4} J_{5}-J_{4} J_{6}\right] \exp \left(J_{5}+J_{6}\right) } \\
& +\left[J_{4} J_{5}-J_{4} J_{6}\right] \exp \left(J_{4}\right)+\left[J_{5} J_{6}-J_{4} J_{5}\right] \exp \left(J_{5}\right)+\left[J_{4} J_{6}-J_{5} J_{6}\right] \exp \left(J_{6}\right),
\end{aligned}
$$




$$
\begin{aligned}
& A_{2}=\left[J_{3} J_{5}-J_{5} J_{6}\right] \exp \left(J_{3}+J_{6}\right)-\left[J_{3} J_{6}-J_{5} J_{6}\right] \exp \left(J_{3}+J_{5}\right)-\left[J_{3} J_{5}-J_{3} J_{6}\right] \exp \left(J_{5}+J_{6}\right) \\
& +\left[J_{3} J_{6}-J_{3} J_{5}\right] \exp \left(J_{3}\right)+\left[J_{3} J_{5}-J_{5} J_{6}\right] \exp \left(J_{5}\right)+\left[J_{5} J_{6}-J_{3} J_{6}\right] \exp \left(J_{6}\right), \\
& A_{3}=\left[J_{4} J_{6}-J_{3} J_{6}\right] \exp \left(J_{3}+J_{4}\right)+\left[J_{3} J_{4}-J_{4} J_{6}\right] \exp \left(J_{3}+J_{6}\right)-\left[J_{3} J_{4}-J_{3} J_{6}\right] \exp \left(J_{4}+J_{6}\right) \\
& -\left[J_{3} J_{4}-J_{3} J_{6}\right] \exp \left(J_{3}\right)-\left[J_{4} J_{6}-J_{3} J_{4}\right] \exp \left(J_{4}\right)-\left[J_{3} J_{6}-J_{4} J_{6}\right] \exp \left(J_{6}\right), \\
& A_{4}=\left[J_{3} J_{5}-J_{4} J_{5}\right] \exp \left(J_{3}+J_{4}\right)+\left[J_{4} J_{5}-J_{3} J_{4}\right] \exp \left(J_{3}+J_{5}\right)-\left[J_{3} J_{5}-J_{3} J_{4}\right] \exp \left(J_{4}+J_{5}\right) \\
& -\left[J_{3} J_{5}-J_{3} J_{4}\right] \exp \left(J_{3}\right)-\left[J_{3} J_{4}-J_{4} J_{5}\right] \exp \left(J_{4}\right)-\left[J_{4} J_{5}-J_{3} J_{5}\right] \exp \left(J_{5}\right), \\
& J_{3}=\frac{\left[J_{1}+\left(J_{1}^{2}+4 \pi^{2}\right)^{1 / 2}\right]}{2}, \quad J_{4}=\frac{\left[J_{1}-\left(J_{1}^{2}+4 \pi^{2}\right)^{1 / 2}\right]}{2}, \quad J_{5}=\frac{\left[J_{2}+\left(J_{2}^{2}+4 \pi^{2}\right)^{1 / 2}\right]}{2}, \\
& J_{6}=\frac{\left[J_{2}-\left(J_{2}^{2}+4 \pi^{2}\right)^{1 / 2}\right]}{2}, \quad g_{1}=\frac{\left[\lambda+\left(\lambda^{2}+4 \pi^{2}+4 M^{2}\right)^{1 / 2}\right]}{2}, \\
& g_{2}=\frac{\left[\lambda-\left(\lambda^{2}+4 \pi^{2}+4 M^{2}\right)^{1 / 2}\right]}{2}, \quad s_{1}=\frac{\left[\lambda \operatorname{Pr}+\left(\lambda^{2} \operatorname{Pr}^{2}+4 \pi^{2}\right)^{1 / 2}\right]}{2}, \\
& s_{2}=\frac{\left[\lambda \operatorname{Pr}-\left(\lambda^{2} \operatorname{Pr}^{2}+4 \pi^{2}\right)^{1 / 2}\right]}{2}, \quad b_{1}=\frac{\lambda}{\left[A\left\{\exp \left(J_{1}\right)-\exp \left(J_{2}\right)\right\}\right]}, \\
& b_{2}=3 J_{1} J_{3}-\lambda J_{3}, \quad b_{3}=3 J_{1} J_{4}-\lambda J_{4}, \quad b_{4}=3 J_{2} J_{5}-\lambda J_{5}, \quad b_{5}=3 J_{2} J_{6}-\lambda J_{6}, \\
& b_{6}=2 J_{2} J_{3}+J_{1} J_{3}-\lambda J_{3}, \quad b_{7}=2 J_{2} J_{4}+J_{1} J_{4}-\lambda J_{4}, \quad b_{8}=2 J_{1} J_{5}+J_{2} J_{5}-\lambda J_{5}, \\
& b_{9}=2 J_{1} J_{6}+J_{2} J_{6}-\lambda J_{6}, \quad C_{1}=\frac{\lambda^{2} \mathrm{Pr}^{2}}{[A\{\exp (\lambda \operatorname{Pr})-1\}]}, \quad C_{2}=J_{1} J_{3}+J_{3} \lambda \operatorname{Pr}, \\
& C_{3}=J_{1} J_{4}+J_{4} \lambda \operatorname{Pr}, \quad C_{4}=J_{2} J_{5}+J_{5} \lambda \operatorname{Pr}, \quad C_{5}=J_{2} J_{6}+J_{6} \lambda \operatorname{Pr}, \\
& C_{6}=\frac{\lambda}{\left[A\left\{\exp \left(J_{1}\right)-\exp \left(J_{2}\right)\right\} \cdot\left\{\exp \left(g_{1}\right)-\exp \left(g_{2}\right)\right\}\right]}, \\
& C_{7}=\frac{\lambda^{2} \operatorname{Pr}^{2}}{\left[A\{\exp (\lambda \operatorname{Pr})-1\} \cdot\left\{\exp \left(s_{1}\right)-\exp \left(s_{2}\right)\right\}\right]}, \\
& E=C_{6}\left[A_{1}\left\{\frac{J_{1}\left(\exp \left(J_{2}+g_{2}\right)-\exp \left(J_{1}+J_{2}+J_{3}\right)\right)}{b_{2}}-\frac{J_{2}\left(\exp \left(J_{1}+g_{2}\right)-\exp \left(J_{1}+J_{2}+J_{3}\right)\right)}{b_{6}}\right\}\right. \\
& +A_{2}\left\{\frac{J_{1}\left(\exp \left(J_{2}+g_{2}\right)-\exp \left(J_{1}+J_{2}+J_{4}\right)\right)}{b_{3}}-\frac{J_{2}\left(\exp \left(J_{1}+g_{2}\right)-\exp \left(J_{1}+J_{2}+J_{4}\right)\right)}{b_{7}}\right\} \\
& -A_{3}\left\{\frac{J_{1}\left(\exp \left(J_{2}+g_{2}\right)-\exp \left(J_{1}+J_{2}+J_{5}\right)\right)}{b_{8}}-\frac{J_{2}\left(\exp \left(J_{1}+g_{2}\right)-\exp \left(J_{1}+J_{2}+J_{5}\right)\right)}{b_{4}}\right\} \\
& -A_{4}\left\{\frac{J_{1}\left(\exp \left(J_{2}+g_{2}\right)-\exp \left(J_{1}+J_{2}+J_{6}\right)\right)}{b_{9}}\right. \\
& \left.\left.-\frac{J_{2}\left(\exp \left(J_{1}+g_{2}\right)-\exp \left(J_{1}+J_{2}+J_{6}\right)\right)}{b_{5}}\right\}\right]
\end{aligned}
$$




$$
\begin{array}{r}
F=C_{6}\left[A _ { 1 } \left\{\frac{J_{1}\left(\exp \left(J_{1}+J_{2}+J_{3}\right)-\exp \left(J_{2}+g_{1}\right)\right)}{b_{2}}\right.\right. \\
\left.-\frac{J_{2}\left(\exp \left(J_{1}+J_{2}+J_{3}\right)-\exp \left(J_{1}+g_{1}\right)\right)}{b_{6}}\right\} \\
+A_{2}\left\{\frac{J_{1}\left(\exp \left(J_{1}+J_{2}+J_{4}\right)-\exp \left(J_{2}+g_{1}\right)\right)}{b_{3}}\right. \\
-A_{3}\left\{\frac{J_{1}\left(\exp \left(J_{1}+J_{2}+J_{5}\right)-\exp \left(J_{2}+g_{1}\right)\right)}{b_{8}}\right. \\
\left.-\frac{J_{2}\left(\exp \left(J_{1}+J_{2}+J_{4}\right)-\exp \left(J_{1}+g_{1}\right)\right)}{b_{7}}\right\} \\
-A_{4}\left\{\frac{J_{1}\left(\exp \left(J_{1}+J_{2}+J_{5}\right)-\exp \left(J_{1}+g_{1}\right)\right)}{b_{4}}\right\} \\
\left.\left.b_{9}\right)-\exp \left(J_{2}+g_{1}\right)\right) \\
\left.\left.-\frac{J_{2}\left(\exp \left(J_{1}+J_{2}+J_{6}\right)-\exp \left(J_{1}+g_{1}\right)\right)}{b_{5}}\right\}\right],
\end{array}
$$$$
R=C_{7}\left[\frac{A_{1}\left\{\exp \left(s_{2}\right)-\exp \left(J_{3}+\lambda \operatorname{Pr}\right)\right\}}{C_{2}}+\frac{A_{2}\left\{\exp \left(s_{2}\right)-\exp \left(J_{4}+\lambda \operatorname{Pr}\right)\right\}}{C_{3}}\right.
$$$$
\left.-\frac{A_{3}\left\{\exp \left(s_{2}\right)-\exp \left(J_{5}+\lambda \operatorname{Pr}\right)\right\}}{C_{4}}-\frac{A_{4}\left\{\exp \left(s_{2}\right)-\exp \left(J_{6}+\lambda \operatorname{Pr}\right)\right\}}{C_{5}}\right],
$$$$
S=C_{7}\left[\frac{A_{1}\left\{\exp \left(J_{3}+\lambda \operatorname{Pr}\right)-\exp \left(s_{1}\right)\right\}}{C_{2}}+\frac{A_{2}\left\{\exp \left(J_{4}+\lambda \operatorname{Pr}\right)-\exp \left(s_{1}\right)\right\}}{C_{3}}\right.
$$$$
\left.-\frac{A_{3}\left\{\exp \left(J_{5}+\lambda \operatorname{Pr}\right)-\exp \left(s_{1}\right)\right\}}{C_{4}}-\frac{A_{4}\left\{\exp \left(J_{6}+\lambda \operatorname{Pr}\right)-\exp \left(s_{1}\right)\right\}}{C_{5}}\right] \text {. }
$$

Now, after knowing the velocity field, we can calculate skin-friction components $\tau_{x}$ and $\tau_{z}$ in the main and transverse directions, respectively, as follows:

$$
\begin{aligned}
& \tau_{x}=\frac{d \tau_{x}^{*}}{\mu U}=\left(\frac{d u_{0}}{d y}\right)_{y=0}+\varepsilon\left(\frac{d u_{11}}{d y}\right)_{y=0} \cos \pi z \\
& \tau_{x}=C_{8}+\varepsilon\left[E g_{1}+F g_{2}\right. \\
&+b_{1}\left\{A_{1}\left(\frac{\left(J_{1}^{2}+J_{1} J_{3}\right) \cdot \exp \left(J_{2}\right)}{b_{2}}-\frac{\left(J_{2}^{2}+J_{2} J_{3}\right) \cdot \exp \left(J_{1}\right)}{b_{6}}\right)\right. \\
&+A_{2}\left(\frac{\left(J_{1}^{2}+J_{1} J_{4}\right) \cdot \exp \left(J_{2}\right)}{b_{3}}-\frac{\left(J_{2}^{2}+J_{2} J_{4}\right) \cdot \exp \left(J_{1}\right)}{b_{7}}\right) \\
& \quad-A_{3}\left(\frac{\left(J_{1}^{2}+J_{1} J_{5}\right) \cdot \exp \left(J_{2}\right)}{b_{8}}-\frac{\left(J_{2}^{2}+J_{2} J_{5}\right) \cdot \exp \left(J_{1}\right)}{b_{4}}\right) \\
&\left.\left.\quad-A_{4}\left(\frac{\left(J_{1}^{2}+J_{1} J_{6}\right) \cdot \exp \left(J_{2}\right)}{b_{9}}-\frac{\left(J_{2}^{2}+J_{2} J_{6}\right) \cdot \exp \left(J_{1}\right)}{b_{5}}\right)\right\}\right] \cos \pi z,
\end{aligned}
$$




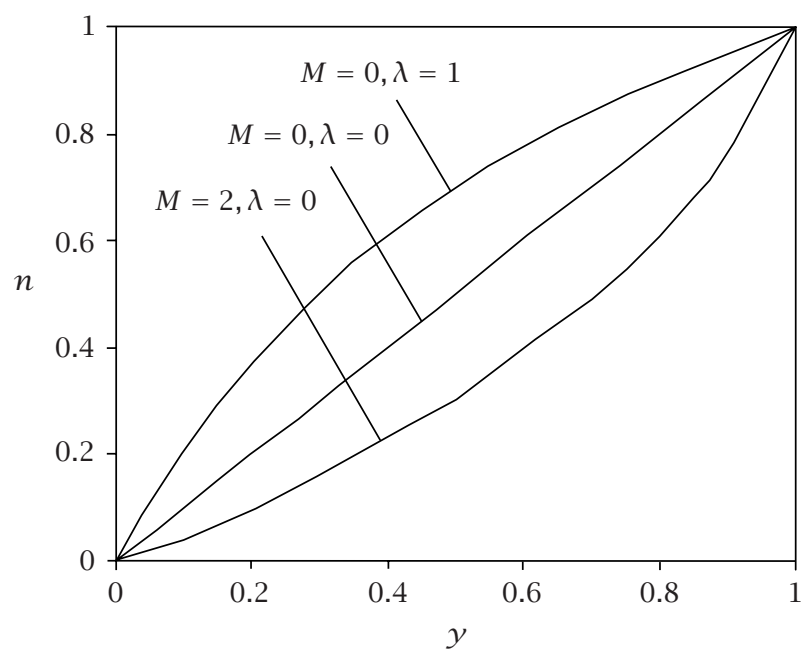

FIGURE 4.1. The velocity profiles for $\varepsilon=0.02$ and $z=0$.

$$
\begin{aligned}
& \boldsymbol{\tau}_{z}=\frac{d \tau_{z}^{*}}{\mu V}=\varepsilon\left(\frac{\partial w_{1}}{\partial y}\right)_{y=0}, \\
& \tau_{z}=-\varepsilon(\pi A)^{-1}\left[A_{1} J_{3}^{2}+A_{2} J_{4}^{2}-A_{3} J_{5}^{2}-A_{4} J_{6}^{2}\right] \sin \pi z .
\end{aligned}
$$

From the temperature field, we can obtain the heat transfer coefficient in terms of Nusselt number as follows:

$$
\begin{gathered}
\mathrm{Nu}=\frac{d q^{*}}{\left[k\left(T_{0}-T_{1}\right)\right]}=\left(\frac{d \theta_{0}}{d y}\right)_{y=0}+\varepsilon\left(\frac{d \theta_{11}}{d y}\right)_{y=0} \cos \pi z, \\
\mathrm{Nu}=C_{9}+\varepsilon\left[R s_{1}+S s_{2}+C_{1}\left\{\frac{A_{1}\left(J_{3}+\lambda \operatorname{Pr}\right)}{C_{2}}+\frac{A_{2}\left(J_{4}+\lambda \operatorname{Pr}\right)}{C_{3}}-\frac{A_{3}\left(J_{5}+\lambda \operatorname{Pr}\right)}{C_{4}}\right.\right. \\
\left.\left.-\frac{A_{4}\left(J_{6}+\lambda \operatorname{Pr}\right)}{C_{5}}\right\}\right] \cos \pi z,
\end{gathered}
$$

where $C_{8}=\left[J_{1} \exp \left(J_{2}\right)-J_{2} \exp \left(J_{1}\right)\right] /\left[\exp \left(J_{1}\right)-\exp \left(J_{2}\right)\right], C_{9}=\lambda \operatorname{Pr} /[\{\exp (\lambda \operatorname{Pr})-1\}]$.

4. Discussion. The main-flow velocity profiles are presented in Figure 4.1. The graph reveals that the main-flow velocity increases with the increase of $M$ (Hartmann number), while a reverse effect is observed with the increase of $\lambda$ (injection/suction). The transverse velocity component is presented in Figure 4.2. It is observed that forward 


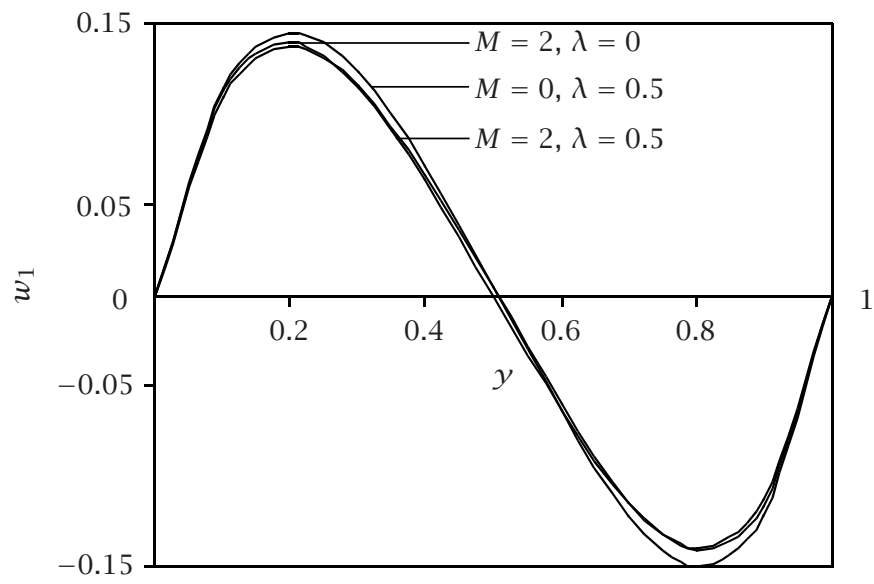

FIGURE 4.2. The transverse velocity components for $z=0.5$.

TABLE 4.1. The values of pressure $\left(p_{1}\right)$ for $z=0$.

\begin{tabular}{rccc}
\hline \multicolumn{1}{c}{$y$} & $\lambda=0.2, M=0$ & $\lambda=0.5, M=0$ & $\lambda=0.5, M=2$ \\
\hline 0 & 22.468 & 8.8780 & 8.9312 \\
0.1 & 15.752 & 6.2182 & 6.3015 \\
0.2 & 10.604 & 4.1773 & 4.2728 \\
0.3 & 6.5114 & 2.5520 & 2.6355 \\
0.4 & 3.0665 & 1.1807 & 1.2316 \\
0.5 & -0.0732 & -0.0773 & -0.0661 \\
0.6 & -3.2202 & -1.3342 & -1.3715 \\
0.7 & -6.6877 & -2.7281 & -2.7983 \\
0.8 & -10.820 & -4.3934 & -4.4752 \\
0.9 & -16.030 & -6.4960 & -6.5628 \\
1.0 & -22.835 & -9.2450 & -9.2758 \\
\hline
\end{tabular}

flow is developed from $y=0$ to about $y=0.5$, and then, onwards, there is backward flow. It is due to the fact that the dragging action of the faster layer exerted on the fluid particles in the neighborhood of the stationary plate is sufficient to overcome the adverse pressure gradient, and hence there is forward flow. The dragging action of the faster layer exerted on the fluid particles will be reduced due to the periodic suction at the upper plate, and hence this dragging action is insufficient to overcome the adverse pressure gradient and there is backward flow. It is noted that this backward flow is just the optical image of the forward flow. Further, it is evident from this figure that 


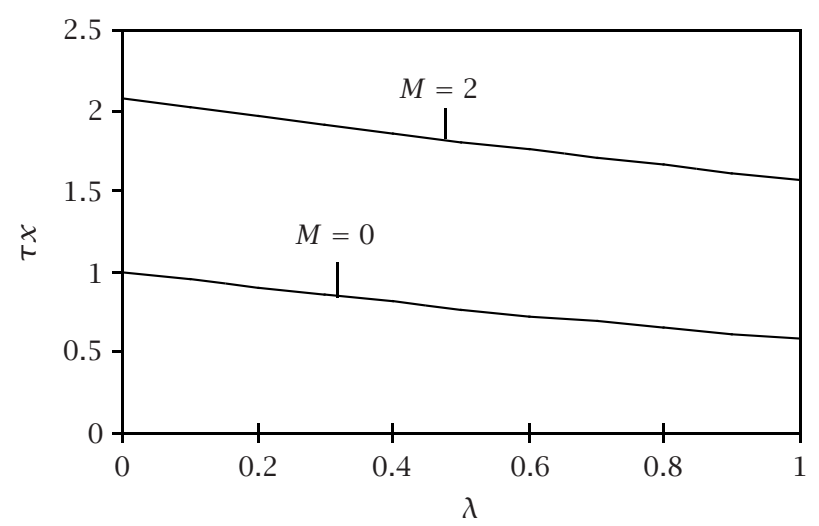

FIGURE 4.3. The values of skin-friction in the main flow direction for $z=0$ and $\varepsilon=0.2$.

TABLE 4.2. The values of skin-friction component $\left(\tau_{z}\right)$ for $\varepsilon=0.2$ and $z=0.5$.

\begin{tabular}{ccc}
\hline$\lambda$ & $M=0$ & $M=2$ \\
\hline 0.1 & 0.35621 & 0.34489 \\
0.2 & 0.35286 & 0.34177 \\
0.3 & 0.34951 & 0.33866 \\
0.4 & 0.34618 & 0.33556 \\
0.5 & 0.34286 & 0.33247 \\
0.6 & 0.33955 & 0.32940 \\
0.7 & 0.33625 & 0.32634 \\
0.8 & 0.33297 & 0.32329 \\
0.9 & 0.32971 & 0.32026 \\
1.0 & 0.32647 & 0.31724 \\
\hline
\end{tabular}

the velocity $w_{1}$ decreases with the increase of $M$ and $\lambda$ both in the forward flow, while a reverse effect is observed in the backward flow. The values of pressure are reported in Table 4.1. It is observed that it decreases with the increase of $\lambda$ and increases with the increase of $M$. Pressure is sufficiently large for small fluid injection/suction.

The values of skin-friction $\tau_{x}$ in the main flow direction are given in Figure 4.3. It is observed that $\tau_{x}$ increases with the increase of $M$ while it decreases with the increase of $\lambda$. The values of skin-friction component $\tau_{z}$ in the transverse direction are presented in Table 4.2. The table shows that $\tau_{z}$ decreases with the increase of both $M$ and $\lambda$.

The values of Nusselt number $(\mathrm{Nu})$ are shown in Figure 4.4. It is observed that $\mathrm{Nu}$ remains almost the same with the increase of $M$, but it decreases with the increase of 


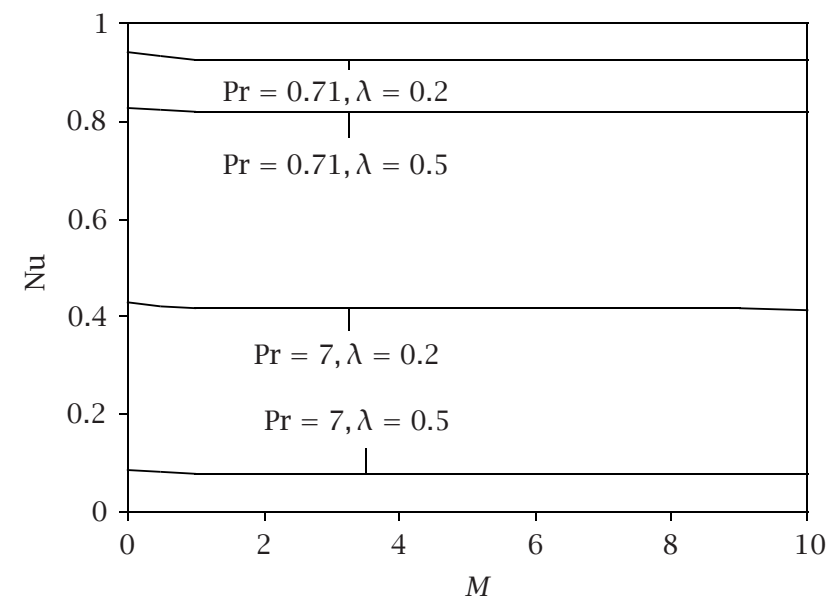

FIGURE 4.4. The values of Nusselt number for $\varepsilon=0.2$ and $z=0$.

$\lambda$ in both situations ( $\operatorname{Pr}=0.71$ (air) and $\operatorname{Pr}=7$ (water)). It is also clear from this figure that $\mathrm{Nu}$ is much lower in the case of water $(\operatorname{Pr}=7)$ than in the case of air $(\operatorname{Pr}=0.71)$.

ACKNOWLEDGMENT. The authors are extremely thankful to the editor and the anonymous reviewers for their valuable suggestions.

\section{REFERENCES}

[1] H. A. Attia and N. A. Kotb, MHD flow between two parallel plates with heat transfer, Acta Mech. 117 (1996), no. 1-4, 215-220.

[2] K. Gersten and J. F. Gross, Flow and heat transfer along a plane wall with periodic suction, Z. Angew. Math. Phys. 25 (1974), 399-408.

[3] H. P. Greenspan and G. F. Carrier, The magnetohydrodynamic flow past a flat plate, J. Fluid Mech. 6 (1959), 77-96.

[4] M. A. Hossain, S. K. Das, and I. Pop, Heat transfer response of MHD free convection flow along a vertical plate to surface temperature oscillations, Internat. J. Non-Linear Mech. 33 (1998), no. 3, 541-553.

[5] V. J. Rossow, On flow of electrically conducting fluids over a flat plate in the presence of a transverse magnetic field, Tech. Report 1358, NACA Ames Aeronautical Laboratory, 1958.

[6] K. D. Singh, Hydromagnetic effects on the three-dimensional flow past a porous plate, Z. Angew. Math. Phys. 41 (1990), no. 3, 441-446.

[7] _ Three-dimensional MHD oscillatory flow past a porous plate, ZAMM Z. Angew. Math. Mech. 71 (1991), no. 3, 192-195.

[8] _ Three-dimensional viscous flow and heat transfer along a porous plate, ZAMM Z. Angew. Math. Mech. 73 (1993), no. 1, 58-61.

[9] _ Three-dimensional Couette flow with transpiration cooling, Z. Angew. Math. Phys. 50 (1999), no. 4, 661-668.

[10] P. Singh, V. P. Sharma, and U. N. Misra, Three dimensional fluctuating flow and heat transfer along a plate with suction, Int. J. Heat Mass Transfer 21 (1978), no. 8, 1117-1123. 
[11]__ Three dimensional free convection flow and heat transfer along a porous vertical plate, Appl. Sci. Res. 34 (1978), no. 1, 105-115.

Pawan Kumar Sharma: Department of Mathematics, University of Rajasthan, Jaipur 302004, India

E-mail address: pawkumar20@yahoo.co.in

R. C. Chaudhary: Department of Mathematics, University of Rajasthan, Jaipur 302004, India E-mail address: chaudhary-rc@uniraj.ernet.in 


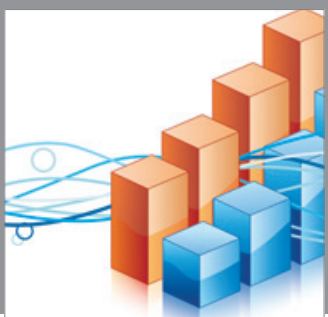

Advances in

Operations Research

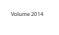

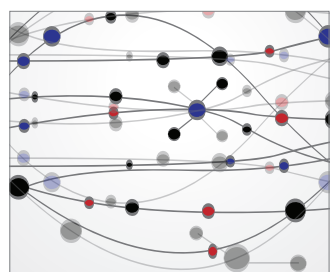

\section{The Scientific} World Journal
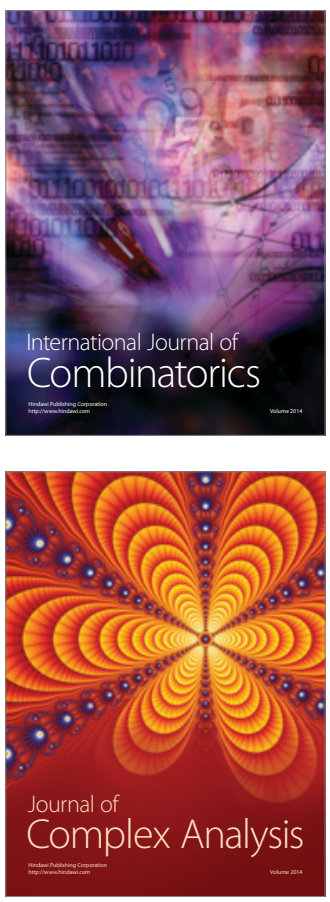

International Journal of

Mathematics and

Mathematical

Sciences
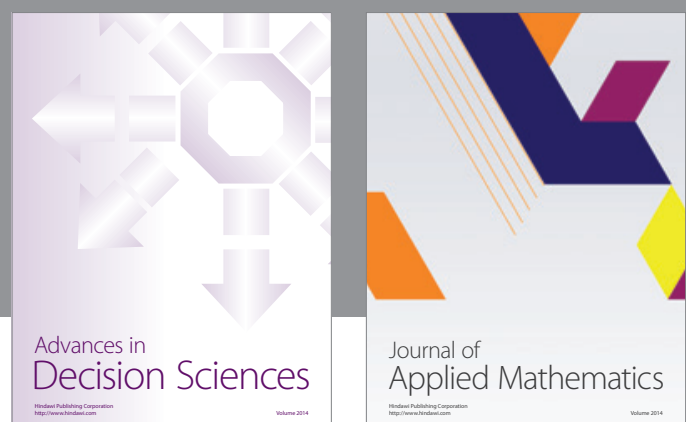

Journal of

Applied Mathematics
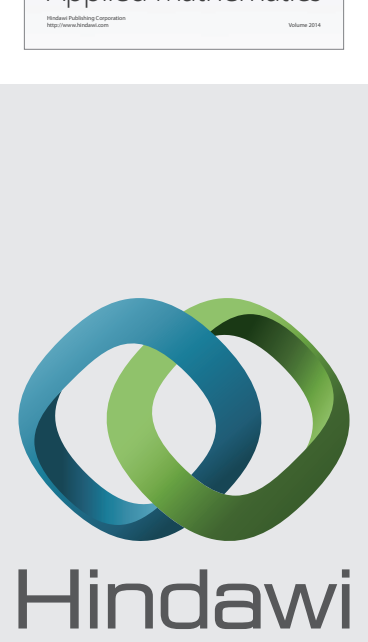

Submit your manuscripts at http://www.hindawi.com
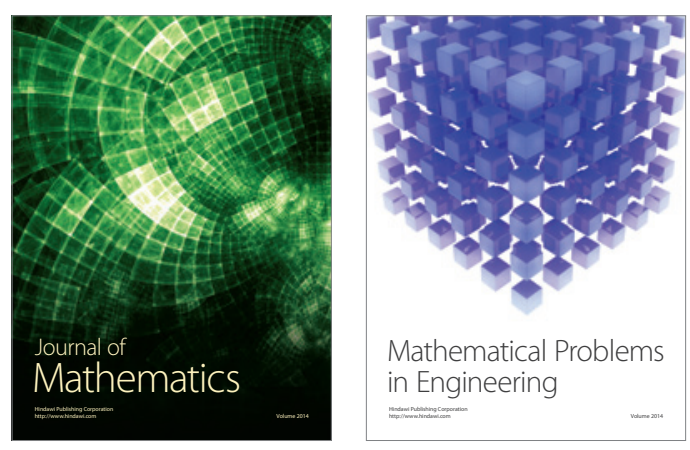

Mathematical Problems in Engineering
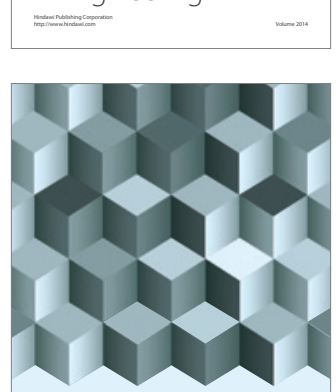

Journal of

Function Spaces
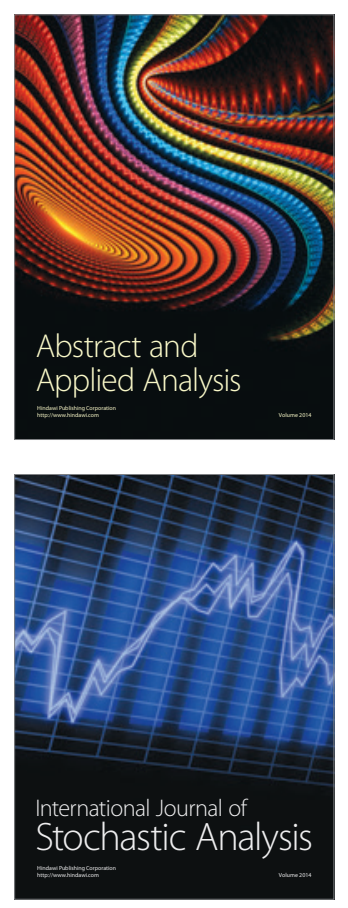

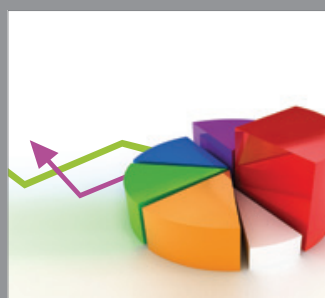

ournal of

Probability and Statistics

Promensencen
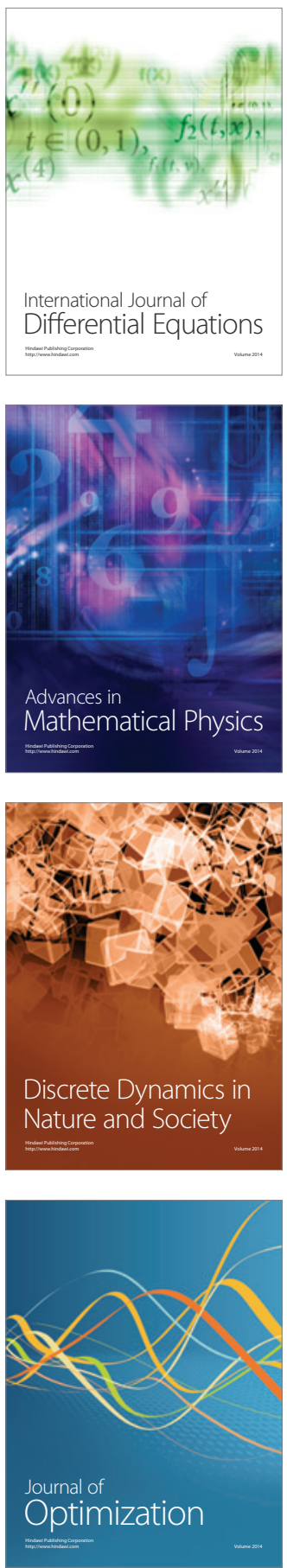